\title{
I General Introduction
}

The idea of a secretory function in the pineal organ has been proposed ever since the time of Galen, but it was not until at the beginning of the nineteenth century, that the gland-like structure of the organ was described (cf. Del Rio Hortega 1932). At the turn of the century clinical investigations were published describing the effect of pineal tumors on various organs e.g. on sexual precocity (Marburg 1909, Pellizi 1910). During the last fifty years a vast amount of literature has been published, in which glandular activity has been described to the mammalian pineal organ, but there is not a single report with clear and substantial evidence for that.

An important new step towards understanding pineal physiology are the reports of Lerner and his co-workers (1958, 1959, 1960), who found in the pineal gland of the cattle a unique compound, $\mathrm{N}$-acetyl-5-methoxy-tryptamine or melatonin, which is $10^{\mathrm{s}}$ times as potent as noradrenaline in causing the aggregation of melanin granules in the amphibian melanocytes. Another important indole-amine derivate, 5-hydroxytryptamine (5-HT) seems to be the precursor of melatonin and also present in large quantities in the pineal gland of various mammals together with a melatonin forming enzyme, hydroxyindole-0-methyl transferase (e.g. Miline et al. 1958, Gíarman and Day 1958, Axelrod et al. 1961, Wurtman et al. 1963). In the rat, the concentration of 5-HT in the pineal gland is higher than in any other organ so far studied. Also other physiologically active amines including noradrenaline and histamine have been found in relatively high concentrations in the pineal gland (e.g. Potter and Axelrod 1963, Machado et al. 1965).

In spite of this new knowledge of the chemistry of the gland there still exists a lot of controversies regarding the function of the gland. The relationship of the pineal gland to various target organs have been extensively explored. The main objective of this research has been to explore the possible influence of the pineal gland on reproduction, on circadian rhytms and on the regulation of aldosterone

secretion. The discovery of melatonin has given, indeed, new evidence for a close correlation between the pineal gland and reproduction, because melatonin is claimed to be a hormone having inhibitory effect on the female gonads (e.g. Wurtman and Axelrod 1965). A number of investigations has been published according to which both 5-HT and melatonin as well as the enzymes participating in their synthesis follow circadian rhytms. These rhytms are supposed to be under the control of the sympathetic nerve fibers of the pineal gland originating from the superior cervical ganglions (e.g. Fiske 1964, Snyder et al. 1964). On the other hand, the effect of the pineal gland on the aldosterone secretion remains unclear. A special stimulating substance, adreno-glomerulotropin has been claimed to be produced by the pineal gland (e.g. Farrell 1959, 1960, Newman Taylor and Farrell 1963), but in many studies this kind of effect could not be reproduced or was situated in nearby diencephalic structures (e.g. Bugnon et al. 1963, Palkovitz et al. 1965). In recent studies, however, 5-HT has been shown to possess aldosterone secretion stimulating effect (e.g. Jouan 1963).

In comparison to the number of investigations, where the influence of the pineal gland on various target organs has been evaluated, fairly few reports have been pulished, in which physiology and biochemistry are related to the structure of the gland although this sort of approach could show precise site of biologically active substances as well as the regulatory mechanism of their synthesis and release inside the gland. The investigations of Owman and his coworkers (1964 a and b) have fruitfully combined experimental conditions and fluorescence microscopic methods for the demonstration of biogenic amines and resulted in localization of 5-HT both in the parenchymal cells and in the sympathetic nerves, of which the latter also contained nor-adrenaline. Numerous attempts have been made to locate the effect of various experimental conditions at an ultrastructural level. However, the results of these studies are highly controversial, which seems to be mainly due to the structural complexity of the gland especially at the fine structural level. 